\title{
The dentate nucleus in Friedreich's ataxia: the role of iron-responsive proteins
}

\author{
Arnulf H. Koeppen · Susan C. Michael • Mitchell D. Knutson • David J. Haile • \\ Jiang Qian · Sonia Levi · Paolo Santambrogio · Michael D. Garrick • \\ Jacques B. Lamarche
}

Received: 1 February 2007 / Revised: 16 March 2007 / Accepted: 16 March 2007 / Published online: 11 April 2007

(C) Springer-Verlag 2007

\begin{abstract}
Frataxin deficiency in Friedreich's ataxia (FRDA) causes cardiac, endocrine, and nervous system manifestations. Frataxin is a mitochondrial protein, and adequate amounts are essential for cellular iron homeostasis. The main histological lesion in the brain of FRDA patients is neuronal atrophy and a peculiar proliferation of synaptic terminals in the dentate nucleus termed grumose degeneration. This cerebellar nucleus may be especially susceptible to FRDA because it contains abundant iron. We examined total iron and selected iron-responsive proteins in the dentate nucleus of nine patients with FRDA and nine normal controls by biochemical and microscopic techniques. Total iron $(1.53 \pm 0.53 \mu \mathrm{mol} / \mathrm{g}$ wet weight) and ferritin $(206.9 \pm 46.6 \mu \mathrm{g} / \mathrm{g}$ wet weight $)$ in FRDA did not significantly differ from normal controls
\end{abstract}

A. H. Koeppen · S. C. Michael - J. B. Lamarche Research, Veterans Affairs Medical Center, Albany, NY, USA

A. H. Koeppen · J. Qian

Neurology, Veterans Affairs Medical Center, Albany, NY, USA

A. H. Koeppen · J. Qian Pathology Service, Veterans Affairs Medical Center, Albany, NY, USA

A. H. Koeppen · J. Qian

Departments of Neurology and Pathology,

Albany Medical College, Albany, NY, USA

M. D. Knutson

Food Science and Human Nutrition Department,

University of Florida, Gainesville, FL, USA

D. J. Haile

Veterans Affairs Medical Center and University of Texas

Health Sciences Center, San Antonio, TX, USA (iron: $1.78 \pm 0.88 \mu \mathrm{mol} / \mathrm{g}$; ferritin: $210.9 \pm 9.0 \mu \mathrm{g} / \mathrm{g}$ ) but Western blots exhibited a shift to light ferritin subunits. Immunocytochemistry of the dentate nucleus revealed loss of juxtaneuronal ferritin-containing oligodendroglia and prominent ferritin immunoreactivity in microglia and astrocytes. Mitochondrial ferritin was not detectable by immunocytochemistry. Stains for the divalent metal transporter 1 confirmed neuronal loss while endothelial cells reacting with antibodies to transferrin receptor 1 protein showed crowding of blood vessels due to collapse of the normal neuropil. Regions of grumose degeneration were strongly reactive for ferroportin. Purkinje cell bodies, their dendrites and axons, were also ferroportin-positive, and it is likely that grumose degeneration is the morphological manifestation of mitochondrial iron dysmetabolism in the terminals

S. Levi · P. Santambrogio

San Raffaele Scientific Institute, Milan, Italy

S. Levi

Vita-Salute San Raffaele University, Milan, Italy

M. D. Garrick

Department of Biochemistry, State University

of New York at Buffalo, Buffalo, NY, USA

J. B. Lamarche

Centre Hospitalier Universitaire de Sherbrooke,

Sherbrooke, QC, Canada

A. H. Koeppen $(\square)$

Neurology Service (528A/127), Stratton VA Medical Center,

113 Holland Ave, Albany, NY 12208, USA

e-mail: arnulf.koeppen@va.gov 
of corticonuclear fibers. Neuronal loss in the dentate nucleus is the likely result of trans-synaptic degeneration.

Keywords Friedreich's ataxia - Atrophy ·

Dentate nucleus · Ferritin · Ferroportin · Iron · Synapses

\section{Introduction}

The mutation in Friedreich's ataxia (FRDA) [3] causes defective transcription of the frataxin gene resulting in moderate to severe loss of frataxin protein. This mitochondrial protein is essential for cellular iron homeostasis though its detailed role in this process has not yet been conclusively established. Friedreich's ataxia is a complex disease, affecting the peripheral and central nervous systems, the heart, and the insulin-producing beta cells of the pancreas. In the brain, the dentate nuclei are almost invariably atrophic. This lesion in the cerebellum of patients with FRDA was at first not fully appreciated because of emphasis on spinal cord and dorsal root ganglia. Atrophy of the dentate nucleus is often severe, combining neuronal loss and a peculiar proliferation of synaptic terminals termed "grumose" degeneration [13-14]. It has become apparent that the severe ataxia of FRDA patients is not solely due to destruction of the ganglia of dorsal spinal roots, the dorsal nuclei of Clarke, and specific long fiber tracts of the spinal cord. Ataxia must also be attributed to dysfunction of the dentate nuclei [24, 25, 30]. Mitochondrial iron excess in FRDA is most apparent in the heart [19, 22], and reduced activity of iron-sulfur cluster-dependent complexes I-III of the electron transport chain, aconitase [26], and ferrochelatase [33] may reflect iron-catalyzed oxidative injury to mitochondria. It is unknown whether the high iron concentration in the human dentate nucleus makes this structure especially vulnerable in FRDA. Several other iron-rich gray matter structures in the brain, such as globus pallidus, substantia nigra, red nucleus, and subthalamic nucleus escape damage in this disease. The study presented here revealed distinct changes in several iron-responsive proteins, that is, ferritin, divalent metal transporter 1 (DMT1), transferrin receptor 1 (TfR1), and ferroportin. Ferroportin [1, 4, 21] is critical in the export of iron from the cytoplasm of many cell types and may be involved in the modification of corticonuclear terminals in grumose degeneration of the dentate nucleus in FRDA.

\section{Materials and methods}

Tissue samples

Fixed and frozen autopsy tissues of nine patients with FRDA were obtained through tissue donations to the National Ataxia Foundation, Minneapolis, MN, USA, and the Friedreich's Ataxia Research Alliance, Alexandria, VA, USA (Table 1). Control samples came from the autopsy practice at the Veterans Affairs Medical Center in Albany, NY, USA. Numerous additional paraffin-embedded samples of cerebellar cortex and dentate nucleus were made available by the Canadian Association of Friedreich's Ataxia Tissue Bank, Department of Pathology, Centre Hospitalier Universitaire de Sherbrooke, Sherbrooke, QC, Canada, and collaborating neuropathologists in the United States, Canada, and Germany. The diagnosis of FRDA was

Table 1 Iron and ferritin concentrations in the dentate nucleus of Friedreich's ataxia

\begin{tabular}{|c|c|c|c|c|c|c|c|}
\hline No. & Sex & $\begin{array}{l}\text { Age of onset } \\
\text { (year) }\end{array}$ & $\begin{array}{l}\text { Age of death } \\
\text { (years) }\end{array}$ & $\begin{array}{l}\text { Allele } 1 \\
\text { (GAA repeats) }\end{array}$ & $\begin{array}{l}\text { Allele } 2 \\
\text { (GAA repeats) }\end{array}$ & $\begin{array}{l}\text { Iron } \\
(\mu \mathrm{mol} / \mathrm{g} \text { wet weight })\end{array}$ & $\begin{array}{l}\text { Ferritin } \\
\text { ( } \mu \mathrm{g} / \mathrm{g} \text { wet weight })\end{array}$ \\
\hline FRDA 1 & $\mathrm{~F}$ & 7 & 38 & 750 & 594 & 0.65 & 210.0 \\
\hline FRDA 2 & $\mathrm{~F}$ & 15 & 33 & 604 & 455 & 1.90 & 201.1 \\
\hline FRDA 3 & $\mathrm{~F}$ & 8 & 23 & 777 & 582 & 1.17 & 280.6 \\
\hline FRDA 4 & M & 16 & 39 & 491 & 370 & 2.40 & 225.8 \\
\hline FRDA 5 & M & 13 & 20 & 850 & 850 & 1.27 & 208.6 \\
\hline FRDA 6 & $\mathrm{~F}$ & 19 & 79 & 549 & 356 & 1.28 & 208.8 \\
\hline FRDA 7 & $\mathrm{~F}$ & 30 & 75 & 631 & 242 & 1.98 & 209.1 \\
\hline FRDA 8 & $\mathrm{~F}$ & 8 & 28 & 709 & 559 & 1.38 & 100.0 \\
\hline FRDA 9 & $\mathrm{~F}$ & 9 & 26 & 850 & 690 & 1.78 & 217.8 \\
\hline All FRDA & - & $7-30$ & $23-79$ & - & - & $1.53 \pm 0.53^{\mathrm{a}}$ & $206.9 \pm 46.6^{\mathrm{a}}$ \\
\hline Normal $^{\mathrm{b}}$ & $\mathrm{M} / \mathrm{F}$ & - & $52-80$ & - & - & $1.78 \pm 0.88^{\mathrm{a}}$ & $210.9 \pm 9.0^{\mathrm{a}}$ \\
\hline Normal $^{\mathrm{c}}$ & $\mathrm{M} / \mathrm{F}$ & - & $30-100$ & - & - & $1.85 \pm 0.87^{\mathrm{a}}$ & - \\
\hline
\end{tabular}

FRDA Friedreich's ataxia, GAA, guanine-adenine-adenine trinucleotide repeats

a Mean $\pm \mathrm{SD},{ }^{\mathrm{b}}$ nine normal controls, ${ }^{\mathrm{c}} 45$ normal dentate nuclei from reference [8] after conversion from $10.35 \mathrm{mg}$ iron/100 g to $1.85 \mu \mathrm{mol} / \mathrm{g}$ wet weight 
based on the classical neuropathological phenotype and confirmation of homozygous GAA expansion in deoxyribonucleic acid extracted from frozen tissue [5] (Table 1).

\section{Biochemical methods}

These techniques were described in detail in a prior publication on FRDA heart [22] and are summarized here as follows: for the assay of total iron, a portion of the dentate nucleus was dissected from sliced cerebellum, weighed, and lyophilized in Kjeldahl flasks. The tissue samples were fully oxidized by heating in $10 \mathrm{M}$ sulfuric acid and hydrogen peroxide. Inorganic iron in the digest was assayed colorimetrically with ferrozine, and results were expressed as $\mu \mathrm{mol}$ iron/g wet weight. For the assay of holoferritin by "double-sandwich" enzyme-linked immunosorbent assay (ELISA), dissected samples of dentate nucleus were homogenized by ultrasonication in $0.05 \mathrm{M}$ Tris buffer $(\mathrm{pH}$ 7.6) containing a protease-inhibitor "cocktail", heat-treated, and centrifuged to obtain a clear supernatant. Polystyrene ELISA plates were coated at $\mathrm{pH} 9.6$ with monoclonal anti-human ferritin (Fitzgerald Industries International, Concord, MA, USA). The supplier of this antibody does not specify the antigenic determinant on the holoferritin molecule. It reacts vigorously with purified human ferritin on double immunodiffusion but not with separated heavy $(H)$ or light $(L)$ ferritin subunits on Western blots. Diluted aliquots of the ferritin-containing supernatants were applied to the pre-coated ELISA plates and allowed to react with the antibody. The next step was application of a rabbit polyclonal anti-ferritin, followed by horseradish peroxidase-conjugated anti-rabbit IgG. After color development with ortho-phenylenediamine and hydrogen peroxide, absorbances at $492 \mathrm{~nm}$ were compared to those of a commercial human heart ferritin standard (Lee Biosolutions, St Louis, MO, USA). Results were expressed as $\mu$ g holoferritin/g wet weight.

For sodium dodecylsulfate-polyacrylamide gel electrophoresis (SDS-PAGE) and Western blotting, the heattreated supernatants were concentrated by centrifugation through ultrafiltration membranes with a molecular cut-off of $100 \mathrm{kDa}$ as described before [22]. After dissociation in sample buffer, they were electrophoresed through gels containing $4 \mathrm{M}$ urea. Proteins were electro-blotted onto polyvinyledene fluoride (PVDF) membranes, and $H$ - and $L$-ferritin subunits were detected immunochemically with a mixture of rabbit anti-human liver ferritin (Immunology Consultant Labs, Newberg, OR, USA) and anti-human $H$-ferritin subunit (Santa Cruz Biotechnology, Santa Cruz, CA, USA). The relative contributions of $H$ - and $L$-ferritin subunits to holoferritin in FRDA and normal dentate gray matter were estimated by densitometry and expressed as an $H / L$ ratio for each specimen.
Immunocytochemistry, lectin affinity cytochemistry, and immunofluorescence

Sections were prepared from paraffin-embedded or sucroseinfiltrated tissue blocks to achieve immunocytochemical detection of ferritin; mitochondrial ferritin; ferroportin; two isoforms of DMT1 [6]; TfR1 (CD71); synaptosome-associated protein 25 (SNAP-25); CD45; glial fibrillary acidic protein (GFAP); mitochondrial complex $\mathrm{V}\left(F_{1} F_{\mathrm{o}}-\beta\right)$; and class III $\beta$-tubulin. Table 2 lists antisera and antibodies, their sources, and embedding, sectioning, and antigen retrieval techniques. Results obtained with anti-CD45 (for microglia) were augmented by lectin affinity cytochemistry with biotinylated Ricinus communis agglutinin 1 (RCA-1) (Table 2). To confirm the specificity of ferroportin immunoreactivity in sections from human brain, two different antibodies raised against mouse ferroportin-specific peptides were available $[1,11]$. Also, diluted anti-ferroportin solutions were preincubated for $4 \mathrm{~h}$ at $4^{\circ} \mathrm{C}$ in a 20 -fold excess (by peptide weight/vol) of a ferroportin-specific peptide [11] or a lysate of human embryonic kidney (HEK) cells transfected to overexpress ferroportin [11]. Lysates of HEK293T cells transfected with empty vector only were used as controls. 2-Mercaptoethanol and SDS in the cell lysates were removed by centrifugation through membranes with a molecular-weight cut-off of 10,000 (Ultrafree-0.5, Millipore, Billerica, MA, USA), followed by washing of the retained proteins with phosphate-buffered saline (PBS). Protein concentrations of diluted antibodies and RCA-1 were optimized empirically to $0.1-10 \mu \mathrm{g} / \mathrm{ml}$. Dewaxing of $6 \mu \mathrm{m}$-thick paraffin sections, oxidation by $3 \%$ hydrogen peroxide in methanol, rehydration, background suppression with $10 \%$ normal horse serum and $4 \%$ bovine serum albumin (BSA), dehydration after completion of immunocytochemical steps, and mounting in xylene-soluble medium followed standard protocols. Positive-contrast immunocytochemistry consisted of the sequential incubation in primary antibody in PBS containing $1 \%$ normal horse serum (by vol) and 4\% BSA (weight/vol), a biotinylated secondary antibody in PBS, and diluted horseradish peroxidase (HRP)-labeled streptavidin in PBS ( $2 \mu \mathrm{g} / \mathrm{ml}$; Sigma, St Louis, MO, USA). For lectin affinity cytochemistry, slides were incubated in biotinylated RCA-1 (0.05 M Tris buffer containing $1 \mathrm{mM} \mathrm{CaCl}$ ), followed by HRP-streptavidin. The final step for immunocytochemistry and lectin affinity cytochemistry was immersion in a solution of diaminobenzidine $(0.05 \%)$ and hydrogen peroxide $(0.015 \%)$ in $0.05 \mathrm{M}$ Tris buffer ( $\mathrm{pH} 7.6)$. Light reaction product was enhanced by a $5 \mathrm{~s}$-exposure of the sections to $1 \%$ osmium tetroxide in PBS (pH 7.4).

Double-label immunofluorescence on vibratome sections has been described in detail elsewhere [17]. Briefly, the $40 \mu \mathrm{m}$-thick floating sections were oxidized with sodium metaperiodate, reduced by sodium borohydride, and 


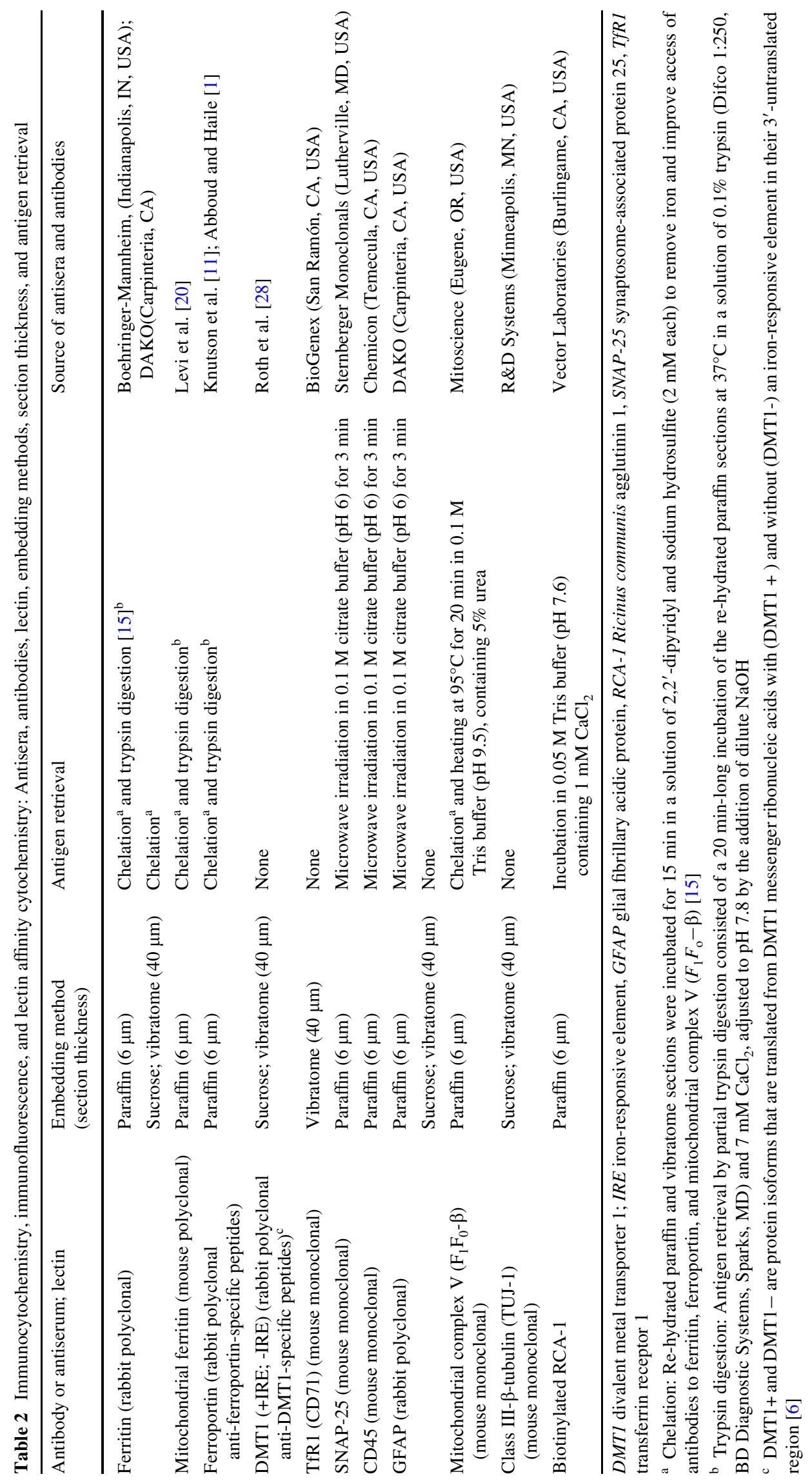


Fig. 1 Macroscopic iron staining of the dentate nucleus. a FRDA, b Normal. Fixed slices of cerebellum were immersed in Perls's solution of $1 \%$ potassium ferrocyanide in $1 \%$ hydrochloric acid. The dentate nucleus in FRDA (a) is much smaller than that in the normal specimen (b). It lacks the distinct gray matter ribbon of the normal nucleus, and the intensity of the iron staining is lower than normal

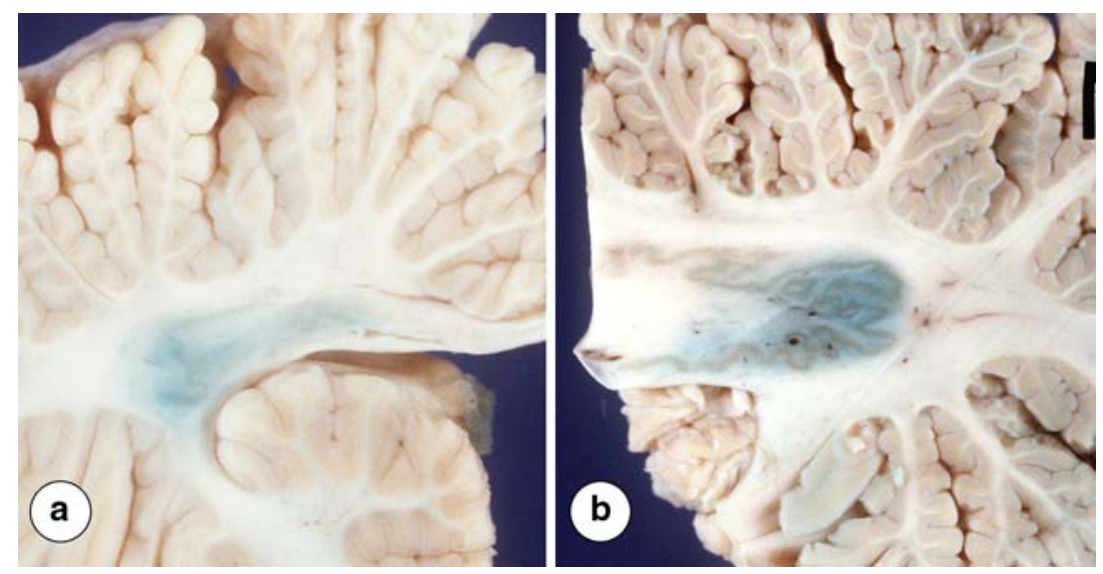

permeabilized with dimethylsulfoxide. Subsequent steps included incubation in "suppressor serum" [10\% normal horse serum (by vol) containing 4\% BSA (weight/vol) and $0.1 \%$ octyl phenol ethoxylate (Triton $\mathrm{X}-100 /$ by vol)], immersion in primary antiserum (antibodies), and application of biotinylated secondary antibodies (to mouse or rabbit $\mathrm{IgG}$, depending on the species of origin of the primary antibody). The sections were then incubated with DyeMerlabeled streptavidin (Molecular Probes, Eugene, OR, USA). DyeMer, the fluorophore of the conjugate, absorbs incident blue laser light maximally at $488 \mathrm{~nm}$ and fluoresces at a peak emission of $605 \mathrm{~nm}$. After washing, the sections were again immersed in suppressor serum and exposed to the second antiserum or antibody, followed by fluorescein isothiocyanate (FITC)-labeled anti-IgG of the species producing the primary antibody. The sections were mounted on glass slides in an aqueous medium (Permafluor; Immunon, Pittsburgh, PA, USA) and viewed in a confocal microscope (Meridian Insight, Okemos, MI, USA). Simultaneous emissions were recorded at filter settings of $530 \pm 30 \mathrm{~nm}$ for FITC and $605 \mathrm{~nm}$ (long pass) for DyeMer.

\section{Results}

\section{Macroscopic iron stains}

Figure 1 shows a comparison of the dentate nuclei in FRDA (Fig. 1a) and in a normal person (Fig. 1b) after macroscopic staining for iron with Perls's reagents (an aqueous solution of $1 \%$ hydrochloric acid and $1 \%$ potassium ferrocyanide). In FRDA, the normal meandering gray matter ribbon of the nucleus is indistinct, and the stain suggests loss of iron.

\section{Biochemical assays}

Quantitative assay of total iron shows no difference between nine FRDA patients and nine normal controls
(Table 1). The iron content in control specimens is identical to the levels reported by Hallgren and Sourander [8]. Table 1 also shows the holoferritin levels in the dentate nucleus in FRDA and normal persons. They are not significantly different but electrophoretic separation of heavy $(H)$ and light $(L)$ ferritin subunits by SDS-PAGE in $4 \mathrm{M}$ urea, and Western blotting, suggest a shift to a higher contribution of $L$-subunits to holoferritin in FRDA when compared with the normal extract (N in Fig. 2). Greater density of the $L$-subunit reaction product is distinctly visible in lanes 3,4 , 7 , and 8 . Lanes 1-8 correspond to FRDA cases $1-8$ in Table 1 but band densities cannot be directly compared with the amounts of holoferritin due to somewhat variable recovery after membrane ultrafiltration (see Materials and methods). In case FRDA 9, insufficient wet tissue was available for SDS-PAGE. Densitometry reveals $H / L$ ratios in normal dentate nucleus that range from 1.29 to 19.7 $[10.5 \pm 6.5$ (mean \pm standard deviation)]. The comparable $H / L$ ratio for FRDA is $2.9 \pm 0.9$ (range 0.58-4.8). The difference is significant by analysis of variance $(\alpha=0.05)$.

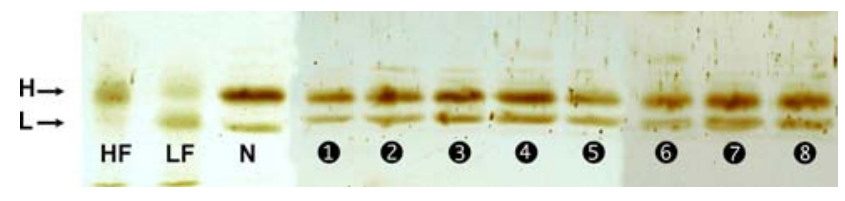

Fig. 2 Western blot of $L$ - and H-ferritin subunits. Ferritin subunits in the extracts of the dentate nucleus of eight FRDA cases were separated by SDS-PAGE in the presence of $4 \mathrm{M}$ urea, electroblotted onto PVDF membranes, and visualized by immunochemical reactions. All sample lanes $(N, 1-8)$ were loaded with extract of the same amount of wet tissue $(2.5 \mathrm{mg})$. The location of the subunits on the blots was identified by comparison with heart ferritin $(H F, \mathrm{H}$-ferritin rich) and liver ferritin ( $L F, L$-ferritin rich). Lane $N$ shows the relative density of the $\mathrm{H}$ - and $L$-units in an extract of a normal dentate nucleus. Lanes $1-8$ correspond to cases $1-8$ in Table 1 . The relative density of $L$-subunits is visibly increased in lanes $3,4,7$, and 8 of the FRDA cases when compared to the normal state $(N)$. Densitometry shows a significant decline of the $\mathrm{H} / L$ ratios in the eight cases of FRDA when compared to nine normal controls 
Immunocytochemistry, immunofluorescence, and lectin affinity cytochemistry

Figure 3a-f illustrates the changes in the cellular localization of ferritin in the dentate nucleus of FRDA patients. The normal dentate nucleus displays cells with intense cytoplasmic ferritin reaction product in juxtaneuronal cells that may be interpreted as oligodendroglia (Fig. 3b). They are nonreactive with anti-CD45 or RCA-1. The dentate nucleus in
FRDA (Fig. 3a) shows severe neuronal loss, and ferritinreactive cells have the morphology, immuno-phenotype (CD45) (Fig. 3a, lower inset), and lectin affinity of microglia (RCA-1) (Fig. 3a, upper inset). Some of the CD45-reactive cells are hypertrophic (Fig. 3a, lower inset). Double-label immunofluorescence in Fig. 3d, f shows the close proximity of ferritin-reactive oligodendroglia (arrows in Fig. 3d, f) to normal neuronal cell bodies. In FRDA (Fig. 3c), the TUJ-1 antibody confirms the total loss of class III- $\beta$-tubulin in the
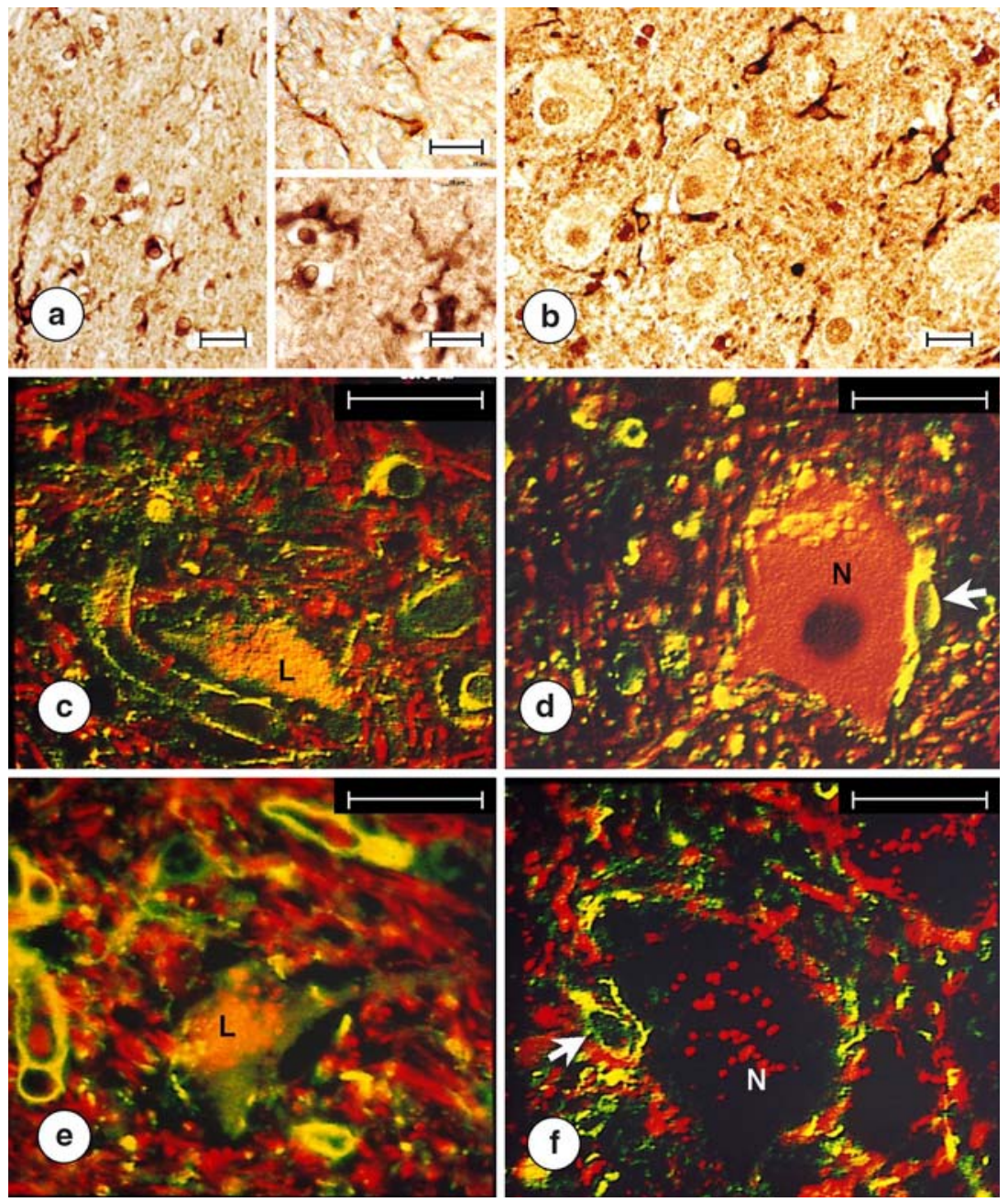

Fig. 3 Immunocytochemistry, lectin affinity cytochemistry, and immunofluorescence of the dentate nucleus in FRDA and normal controls. Left panel (a, c, e), FRDA; right panel $(\mathbf{b}, \mathbf{d}, \mathbf{f})$, normal. Immunocytochemistry and lectin affinity cytochemistry: Ferritin-reactive cells have the morphology of microglia (a). The upper inset in a displays lectin (RCA-1) affinity in microglia; the lower inset in a shows microglial hypertrophy in two cells that are immunoreactive with antiCD45. Ferritin immunoreactivity in the normal dentate nucleus occurs mostly in juxtaneuronal oligodendroglia (b). These cells are RCA-1 and CD45 negative (not illustrated). Double-label confocal immunofluorescence for class III $\beta$-tubulin and ferritin: Antibody TUJ-1 to class III $\beta$-tubulin shows a normal neuron $(N)$ in an intense red color and ferritin as green or yellow reaction product. The arrow points to a juxtaneuronal ferritin-reactive oligodendroglial cell (d). In FRDA,

only lipofuscin (" $L$ " in $\mathbf{c}$ ) remains as an indicator of a nerve cell. This neuron is negative with TUJ-1 and lacks juxtaneuronal ferritin-positive oligodendroglia. Ferritin reaction product outlines vessel walls in (c). The normal neuropil of the dentate nucleus displays not ferritin reaction product in vessel walls (d). Double-label immunofluorescence of GFAP and ferritin: In e, an atrophic nerve cell with abundant lipofuscin $(L)$ is surrounded by numerous GFAP-positive astrocytic processes (red), and vessel walls display the orange color of superimposed GFAP (red) and ferritin (green). In the normal state (f), fewer astrocytic processes are present $(r e d)$. They show no ferritin immunoreactivity. Ferritin is confined to a cell (arrow) and delicate processes in close proximity to an intact neuron $(N)$. $L$ lipofuscin; $N$ neuron; a, b, paraffin sections; $\mathbf{c}-\mathbf{f}$ vibratome sections. Magnification markers: $\mathbf{a}$ and insets, $20 \mu \mathrm{m}$; b $20 \mu \mathrm{m}$; $\mathbf{c}-\mathbf{f} 25 \mu \mathrm{m}$ 
cytoplasm of one nerve cell that is still recognizable by its lipofuscin ( $L$ in Fig. 3c). Axons remain highly immunoreactive with TUJ-1 (Fig. 3c). Ferritin reaction product also outlines vessel walls (Fig. 3c), which is not the case in the normal dentate nucleus (Fig. 3d). Figure 3e, f combines images of ferritin (FITC, green) and GFAP (DyeMer, red). The image in Fig. 3e confirms gliosis of the dentate nucleus in FRDA and co-localization of GFAP and ferritin (yellow merged image) in close apposition to vessel walls. In the normal dentate nucleus, GFAP reaction product is less prominent (Fig. 3f), and co-localization with ferritin around blood vessels is absent. The dentate nucleus in FRDA or the normal state yielded no reaction product with polyclonal anti-mitochondrial ferritin [20].

Figure 4 shows ferroportin immunoreactivity in the cerebellar cortex (Fig. 4a1, a2, b1, b2), the white matter of the folia (Fig. 4c, d), and the dentate nucleus (Fig. 4e, f). Preincubation of the antiserum with a ferroportin-specific peptide [11] or lysates of HEK239T cells over-expressing ferroportin [11] eliminated the reaction. Lysates of HEK239T cells transfected with empty vector had no effect on the final reaction product. In promptly fixed tissue, antiferroportin visualizes Purkinje cell perikarya and dendrites, including spiny branchlets. There are no obvious differences in the dendrites of Purkinje cells in FRDA (Fig. 4a1) when compared to the normal dentate nucleus (Fig. 4b1). In
FRDA, the granular layer shows occasional axonal expansions (Fig. 4a2). Purkinje cell axons display dense reaction product (Fig. 4b2). The white matter of the cerebellar folia in FRDA and the normal state contain abundant ferroportin-reactive axons (Fig. 4c, d). The most dramatic abnormality in FRDA is grumose degeneration of the dentate nucleus (Fig. 4e). Neurons are indistinct, and ferroportin reaction product occurs in axons and irregular clusters (Fig. 4e). In contrast, the normal dentate nucleus shows ferroportin reaction product in delicate synaptic terminals abutting neurons (Fig. 4f). Figure 5a-f provides additional immunocytochemical stains of grumose degeneration. The clusters are reactive with anti-SNAP-25 (Fig. 5c) and antimitochondrial complex $\mathrm{V}\left(F_{\mathrm{o}} F_{1}-\beta\right)$ (Fig. 5e). All stains show the disorganization of synaptic terminals undergoing grumose degeneration in FRDA. Though ferroportin and SNAP-25 immunoreactivity in grumose degeneration is high, Fig. 5a, c also suggests an overall depletion of these proteins in the neuropil between the patches of dense reaction product.

Grumose degeneration does not always affect the entire dentate nucleus. It is most abundant when at least some neuronal cell bodies persist (arrow in Fig 5a), and may be absent in cases with total neuronal loss.

Antisera to DMT1+ and DMT1- confirmed neuronal loss in the dentate nucleus in FRDA patients (not illustrated).
Fig. 4 Ferroportin immunocytochemistry in cerebellar cortex and dentate nucleus. Left panel (a1, a2, c, e), FRDA; right panel (b1, b2, d, f), normal. Ferroportin reaction product is present in Purkinje cell perikarya and dendrites, including spiny branchlets in FRDA (a1) and normal cerebellar cortex (b1). a2 shows a ferroportin-positive axonal torpedo in the granular layer in FRDA. b2 The arrow indicates a ferroportin-reactive axon that arises from a Purkinje cell. Many white matter axons are ferroportin reactive (c FRDA, d normal). In the dentate nucleus of FRDA, multiple clusters of grumose degeneration display ferroportin reaction product (e). Neurons are absent. In the normal state (f), neurons are surrounded by delicate ferroportin-reactive terminals supporting their origin from Purkinje cells. Paraffin sections; magnification markers: (a1-b2) $50 \mu \mathrm{m}$; (c-f) $20 \mu \mathrm{m}$
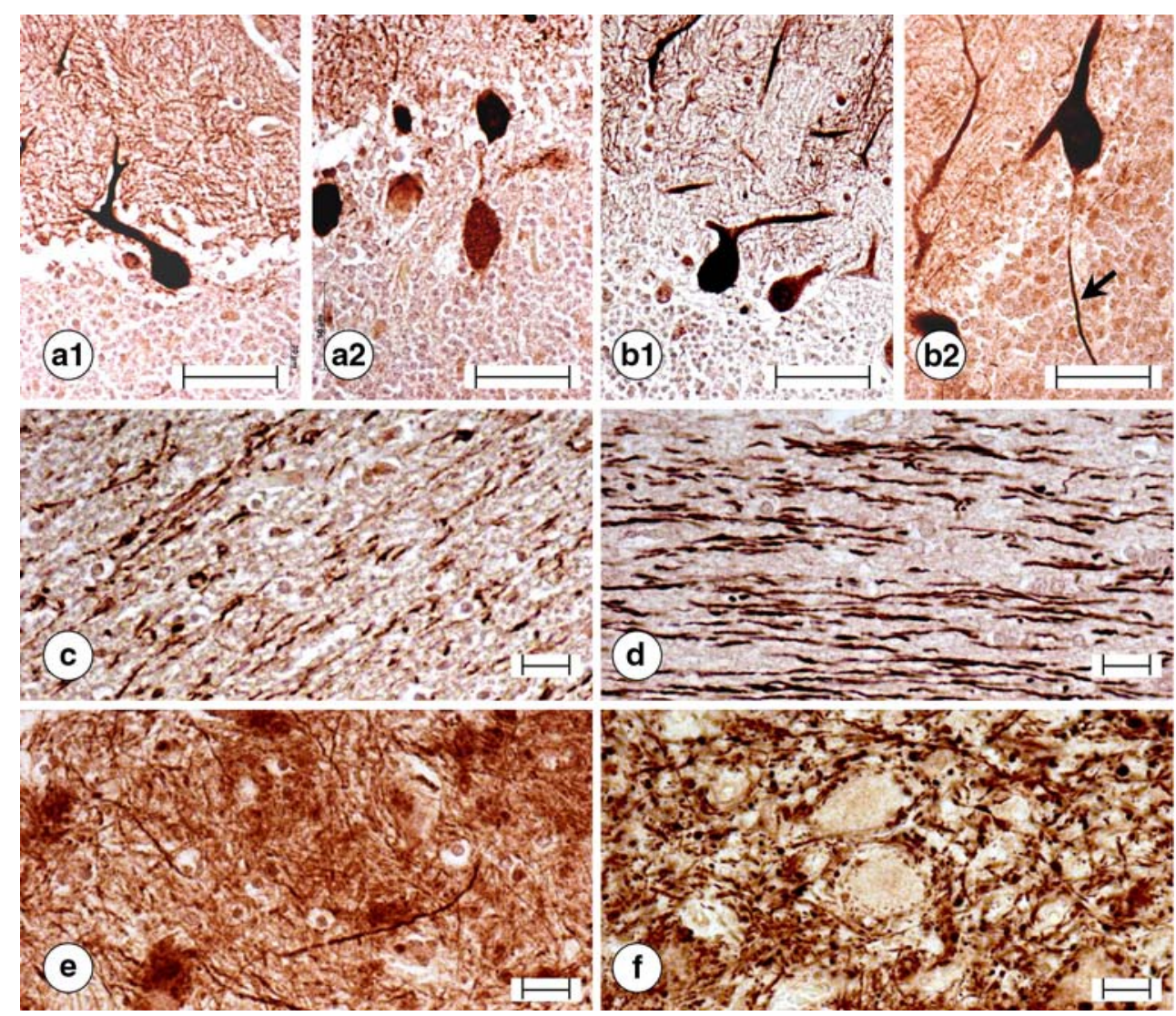


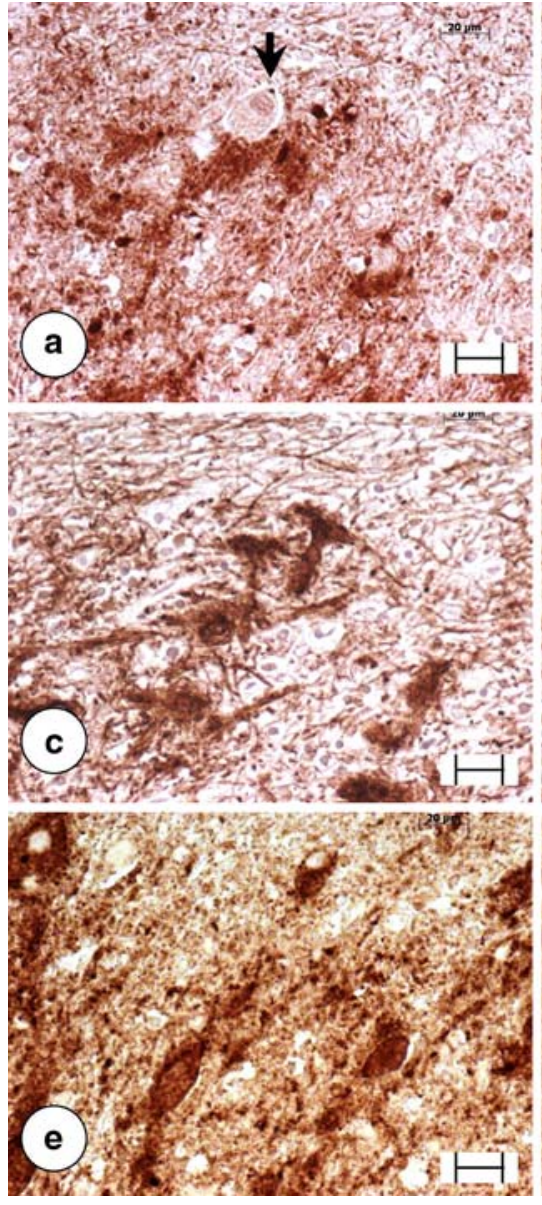

Fig. 5 Immunostaining of grumose degeneration in FRDA. Left panel (a, c, e), FRDA; right panel (b, d, f), normal. a, b ferroportin; c, d SNAP-25; e, f mitochondrial complex V $\left(F_{0} F_{1}-\beta\right)$. All stains show severe disorganization of the dentate nucleus in FRDA (a, c, e) when compared to the normal state $(\mathbf{b}, \mathbf{d}, \mathbf{f})$. Multiple clusters of grumose degeneration in a are ferroportin-reactive, and the largest cluster lies adjacent to a recognizable neuron (arrow). The clusters are also strongly reactive for SNAP-25 (c) and mitochondrial complex V (e). The

Immunoreactivity of TfR 1 showed crowding of blood vessels in the atrophic nucleus that was attributed to shrinkage of the neuropil (not illustrated).

\section{Discussion}

Iron and ferritin in the dentate nucleus of patients with FRDA

The dentate nucleus belongs to the iron-rich structures of the brain [29] but sections of tissues obtained at autopsy and stained by Perls's method do not provide adequate cellular localization of soluble non-heme iron. The reason is delayed fixation and release of iron from the main iron-carrying protein, ferritin, during the interval between death and immersion in formalin. In animal tissues fixed by transcardiac

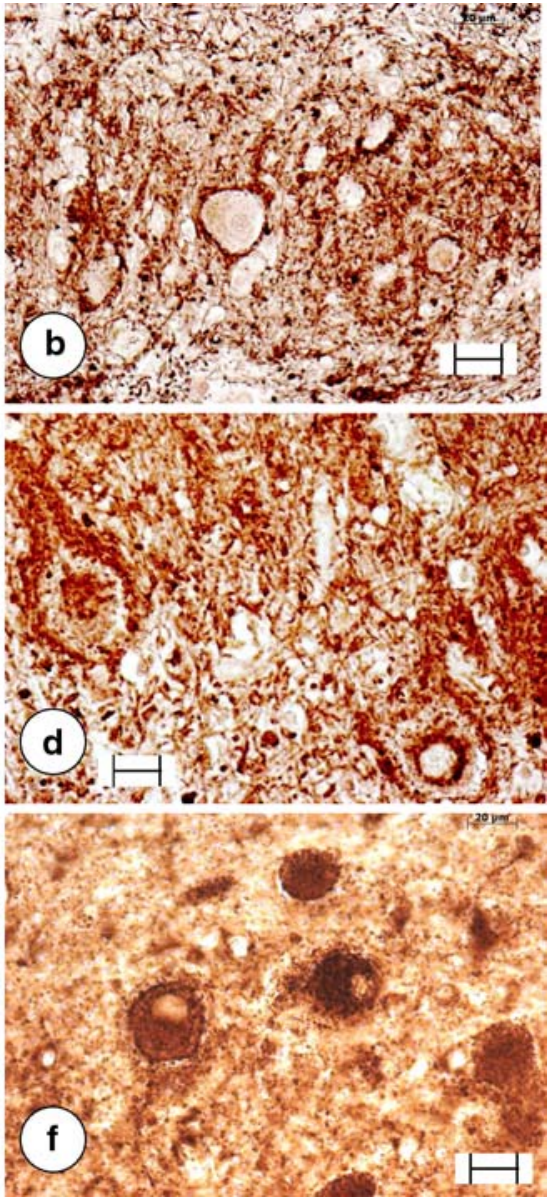

normal dentate nucleus $(\mathbf{b}, \mathbf{d}, \mathbf{f})$ shows ferroportin in terminals about intact neurons and as granular reaction product throughout the normal neuropil (b). SNAP-25 is present in densely packed axosomatic and axodendritic terminals (d). The immunostain for mitochondrial complex V shows dense reaction product in neuronal perikarya and perineuronal granules that likely represent mitochondria in synaptic terminals (f). Paraffin sections; magnification markers, (a-f) $20 \mu \mathrm{m}$

perfusion with aldehydes, iron and ferritin show a similar cellular distribution. Ferritin is a large and relatively stable protein that resists autolytic destruction, and processing into paraffin sections does not destroy its antigenic determinants. In the normal human dentate nucleus, ferritin is concentrated in juxtaneuronal cells (Fig. 3b, d) that lack affinity for RCA-1 and anti-CD45, excluding their derivation from microglia. They have the appearance of satellite oligodendroglia and disappear when neurons become atrophic in FRDA (Fig. 3c, e). The loss of these cells coincides with the shift of ferritin immunoreactivity to microglia (Fig. 3a) and astrocytic foot plates (Fig. 3c, e). Oligodendroglia in the adjacent white matter are also intensely ferritin-positive but this immunoreactivity does not change as FRDA progresses.

The results of total iron and holoferritin assays do not provide insight into the potential role of iron in the pathogenesis 
of the FRDA-related lesion in the dentate nucleus. The shift to $L$-ferritin subunits is non-specific, and the observations only confirm what is known about general iron-handling in mammalian tissues. Macro-staining for iron suggests a net loss of the metal (Fig. 1a) but quantitative assays do not confirm reduced levels. Obviously, the dentate nucleus affected by FRDA retains total iron though it relocates it to ferritin in microglia and astrocytes.

Immunocytochemical detection of ferritin has been used for the identification of microglia in various human brain disorders [10, 15] but is probably less useful for "resting" microglia. The number of microglia in sections of the normal human dentate nucleus is variable [16] but greater abundance (Fig. 3a, upper inset) and hypertrophy (Fig. 3a, lower inset) are probably associated with rapidly progressive FRDA. The declining $H / L$ ferritin subunit ratios in the dentate nucleus in FRDA are the likely result of $H$-ferritin subunit loss in oligodendroglia and increased $L$-ferritin subunit biosynthesis in microglia. A similar shift to $L$-subunits occurs in heart holoferritin of patients with FRDA [22].

Ferroportin in the dentate nucleus

In extracerebral tissues, ferroportin is now recognized as a key protein in the export of iron from duodenal epithelial cells, hepatocytes, and macrophages. It cannot be assumed that this iron-carrying protein serves a similar function in Purkinje cells and their processes (Fig. 4). Grumose degeneration of the dentate nucleus also occurs in two other common diseases of the CNS that are very different from FRDA, namely, spinocerebellar ataxia type 3 (SCA-3)/ Machado-Joseph disease (MJD) and progressive supranuclear palsy (PSP) [9, 13-14]. We examined ferroportin immunoreactivity of grumose degeneration in three cases of SCA-3/MJD and four cases of PSP. As expected, Purkinje cells, corticonuclear fibers, terminals, and regions of grumose degeneration were ferroportin-reactive but grumose degeneration in these diseases lacked the elaborate ferroportin-reactive clusters of FRDA (Figs. 4e, 5a). Wu et al. [32] localized ferroportin to synaptic vesicles by immuno-electron microscopy, and their work is of immediate interest to the pathogenesis of grumose degeneration. The authors suggested that the presence of an IRE in the 5 '-UTR of ferroportin mRNA makes synapses vulnerable to iron toxicity. Neurons [2] and certain cells of the retina [7] may utilize iron-responsive ferroportin translation as a mechanism to eliminate iron toxicity. Synaptic terminals contain abundant mitochondria, and the strong immunoreactivity of ferroportin in grumose degeneration of FRDA may be an indicator of mitochondrial iron dysmetabolism as reported for FRDA heart [22].

We propose that local iron excess in or near mitochondria increases ferroportin biosynthesis by a translational mechanism involving iron-regulatory proteins and an IRE in the $5^{\prime}$-UTR of the cognate mRNA. Under this assumption, ferroportin is a putative marker for frataxin deficiency in synaptic terminals of corticonuclear cerebellar fibers. Ferroportin is probably not relevant to the pathogenesis of grumose degeneration in SCA-3/MJD and PSP.

Why do other iron-rich nuclei in the CNS not undergo changes in FRDA? Sections of globus pallidus, substantia nigra, red nucleus, and subthalamic nucleus show no ferroportin reaction product in perikarya or axons. Finely granular reaction product occurs in the neuropil and may be located in synaptic terminals, resembling the positive staining of corticonuclear terminals in the normal dentate nucleus. It is possible that the greater concentration of ferroportin in Purkinje cell-derived terminals makes them more susceptible to iron dysmetabolism than synapses in other CNS locations. The lack of reaction product in the perikarya of neurons other than Purkinje cells in the human CNS is at variance with observations in optimally fixed rat brain [23]. This discrepancy awaits clarification.

Trans-synaptic neuronal atrophy of the dentate nucleus

Over $60 \%$ of all corticonuclear cerebellar fibers derive from Purkinje cells, and atrophy of the dentate nucleus in FRDA and other conditions displaying grumose degeneration may be due to trans-synaptic atrophy. Though these clusters represent a peculiar and abnormal abundance of terminals, the overall density of synapses in the dentate nucleus of patients with FRDA and SCA-3/MJD is lower than normal [18]. Primary Purkinje cell depletion in familial cortical cerebellar atrophy readily explains synaptic loss in the dentate nucleus. In contrast, loss of synaptic terminals in FRDA and SCA-3/MJD cannot be attributed to primary Purkinje cell atrophy because the cerebellar cortex in these ataxias is often entirely normal. Preservation of the inferior olivary nuclei in FRDA and SCA-3/MJD confirms that climbing fibers have normal targets, namely proximal Purkinje cell dendrites. Why do Purkinje cells remain intact while their terminals in the dentate nucleus degenerate? It is known that axonal destruction does not necessarily cause retrograde atrophy of Purkinje cells. These neurons have much greater inherent resistance to axonal interruption than other nerve cells whose efferent fibers begin and end within the CNS [27]. The morphological preservation of Purkinje cells in FRDA does not imply functional integrity, and the deafferentation of the dentate nucleus points to a primary problem in Purkinje cells. In some cases of FRDA, these nerve cells of the cerebellar cortex are abnormal, perhaps as a small signal that a limited retrograde response to axonal interruption does occur [12]. A logical extension of this work is the search for ferritin aggregates at the ultrastructural level in the mitochondria of Purkinje cells and their 
axons, and in regions of grumose degeneration. Bismuth subnitrate enhancement of ferritin showed mitochondrial inclusions with high electron density in cardiac mitochondria of patients with FRDA [22], and this technique may be equally suitable for brain tissue.

Iron as an in-vivo biomarker of FRDA

The normal total iron levels in the dentate nucleus in FRDA reported here must be reconciled with magnetic resonance measurements during life [31]. Waldvogel et al. [31] reported that this neuro-imaging technique suggests a quantitative iron increase in the dentate nucleus of patients with FRDA. Magnetic resonance imaging (MRI) may be misleading because signal depression due to the paramagnetic effect of iron may also detect shifts of the metal into a different state, such as ferritin and hemosiderin, and into more compact tissue in the course of atrophy (Fig. 1a). The same amount of iron in a much thinner dentate nucleus will cause a higher local concentration of the metal and presumably a greater signal depression on T2-weighted images. Nevertheless, MRI may become a useful monitor for individual FRDA patients who receive experimental therapy.

Acknowledgments Dr. Koeppen's laboratory is supported, in part, by the Office of Research and Development (Laboratory Medicine R\&D Service) of the Department of Veterans Affairs, Washington, DC, USA. The authors received financial support from Friedreich's Ataxia Research Alliance, Alexandria, VA, USA (AHK); National Ataxia Foundation, Minneapolis, MN, USA (AHK); Neurochemical Research, Inc., Glenmont, NY, USA (AHK); and NIH grants R01DLK59794 (MDG) and DK065064 (MDK).

\section{References}

1. Abboud S, Haile DJ (2000) A novel mammalian iron-regulated protein involved in intracellular iron metabolism. J Biol Chem 275:19906-19912

2. Aguirre P, Mena N, Tapia V, Arrendondo M, Nuñez MT (2005) Iron homeostasis in neuronal cells: a role for IREG1. BMC Neurosci $6: 3$

3. Campuzano V, Montermini L, Molto MD, Pianese L, Cossee M, Cavalcanti F, Monros E, Rodius F, Duclos F, Monticelli A, Zara F, Canizares J, Koutnikova H, Bidichandani SI, Gellera C, Brice A, Trouillas P, De Michele G, Filla A, De Frutos R, Palau F, Patel PI, Di Donato S, Mandel JL, Cocozza S, Koenig M, Pandolfo M (1996) Friedreich's ataxia: autosomal recessive disease caused by an intronic GAA triplet repeat expansion. Science 271:1423-1427

4. Donovan A, Brownlie A, Zhou Y, Shepard J, Pratt SJ, Moynihan J, Paw BH, Drejer A, Barut B, Zapata A, Law TC, Brugnara C, Lux SE, Pinkus GS, Pinkus JL, Kingsley PD, Palis J, Fleming MD, Andrews NC, Zon LI (2000) Positional cloning of zebrafish ferroportin1 identifies a conserved vertebrate iron exporter. Nature 403:776-781

5. Filla A, De Michele G, Cavalcanti F, Pianese L, Monticelli A, Campanella G, Cocozza S (1996) The relationship between trinucleotide (GAA) repeat length and clinical features in Friedreich ataxia. Am J Hum Genet 59:554-560
6. Garrick MD, Dolan KG, Horbinski C, Ghio AJ, Higgins D, Porubcin M, Moore EG, Hainsworth LN, Umbreit JN, Conrad ME, Feng L, Lis A, Roth JA, Singleton S, Garrick LM (2003) DMT1: A mammalian transporter for multiple metals. BioMetals 16:41-54

7. Hahn P, Dentchev T, Qian Y, Roault F, Harris ZL, Dunaief JL (2004) Immunolocalization and regulation of iron handling proteins ferritin and ferroportin in the retina. Mol Vis 10:598-607

8. Hallgren B, Sourander P (1958) The effect of age on the non-haemin iron in the human brain. J Neurochem 3:41-51

9. Ishizawa K, Lin W-L, Tiseo P, Hone WG, Davies P, Dickson DW (2000) A qualitative and quantitative study of grumose degeneration in progressive supranuclear palsy. J Neuropathol Exp Neurol 59:513-524

10. Kaneko Y, Kitamoto J, Tateishi J, Yamaguchi K (1989) Ferritin immunohistochemistry as a marker for microglia. Acta Neuropathol (Berlin) 79:129-136

11. Knutson MD, Oukka M, Koss LM, Aydemir FD, WesslingResnick M (2005) Iron release from macrophages after erythrophagocytosis is up-regulated by ferroportin 1 overexpression and down-regulated by hepcidin. Proc Natl Acad Sci USA 102:13241328

12. Koeppen AH (1991) The Purkinje cell and its afferents in human hereditary ataxia. J Neuropathol Exp Neurol 50:505-514H

13. Koeppen AH (1998) The hereditary ataxias. J Neuropathol Exp Neurol 57:531-543

14. Koeppen AH (2002) Neuropathology of the inherited ataxias. In: Manto M-U, Pandolfo M (eds) The cerebellum and its disorders. Cambridge University Press, Cambridge, pp 387-405

15. Koeppen AH, Dentinger MP (1988) Brain hemosiderin and superficial siderosis of the central nervous system. J Neuropathol Exp Neurol 47:249-270

16. Koeppen AH, Turok DI (1992) The cerebellar cortex and the dentate nucleus in hereditary ataxia. In: Plaitakis A (ed) Cerebellar degenerations: clinical neurobiology. Kluwer, Boston, pp 205 236

17. Koeppen AH, Dickson AC (2002) Tin-protoporphyrin prevents experimental superficial siderosis in rabbits. J Neuropathol Exp Neurol 61:689-701

18. Koeppen AH, Dickson AC, Lamarche JB, Robitaille Y (1999) Synapses in the hereditary ataxias. J Neuropathol Exp Neurol 58:748-764

19. Lamarche JB, Côté M, Lemieux B (1980) The cardiomyopathy of Friedreich's ataxia. Morphological observations in 3 cases. Can J Neurol Sci 7:389-396

20. Levi S, Corsi B, Bosisio M, Invernizzi R, Volz A, Sanford D, Arosio P, Drysdale J (2001) A human mitochondrial ferritin encoded by an intronless gene. J Biol Chem 276:24437-24440

21. McKie AT, Marciani P, Rolfs A, Brennan K, Wehr K, Barrow D, Miret S, Bomford A, Peters TJ, Farzaneh F, Hediger MA, Hentze MW, Simpson RJ (2000) A novel duodenal iron-regulated transporter, IREG1, implicated in the basolateral transfer of iron to the circulation. Mol Cell 5:299-309

22. Michael S, Petrocine SV, Qian J, Lamarche JB, Knutson MD, Garrick MD, Koeppen AH (2006) Iron and iron-responsive proteins in the cardiomyopathy of Friedreich's ataxia. Cerebellum 5:257-267

23. Moos T, Rosengren Nielsen T (2006) Ferroportin in the postnatal rat brain: Implications for axonal transport and neuronal export of iron. Semin Pediatr Neurol 13:149-157

24. Mott FW (1907) Case of Friedreich's disease, with autopsy and systematic microscopical examination of the nervous system. Arch Neurol Psychiatr 3:180-200

25. Oppenheimer DR (1979) Brain lesions in Friedreich's ataxia. Can J Neurol Sci 6:173-176

26. Rötig A, de Lonlay P, Chretien D, Foury F, Koenig M, Sidi D, Munnich A, Rustin P (1997) Aconitase and mitochondrial iron-sulphur protein deficiency in Friedreich ataxia. Nat Genet 17:215-217 
27. Rossi F, Gianola S, Corvetti L (2006) The strange case of Purkinje axon regeneration and plasticity. Cerebellum 5:174-182

28. Roth JA, Horbinski C, Feng L, Dolan KG, Higgins D, Garrick MD (2000) Differential localization of divalent metal transporter 1 with and without iron response element in rat PC12 and sympathetic neuronal cells. J Neurosci 20:7595-75601

29. Spatz H (1922) Über den Eisennachweis im Gehirn, besonders in Zentren des extrapyramidal-motorischen Systems. Z Ges Neurol Psychiat 77:261-390

30. Urich H, Normal RM, Lloyd OC (1957) Suprasegmental lesions in Friedreich's ataxia. Confin Neurol 17:360-371
31. Waldvogel D, van Gelderen P, Hallett M (1999) Increased iron in the dentate nucleus of patients with Friedreich's ataxia. Ann Neurol 46:123-125

32. Wu LJ, Leenders AG, Cooperman S, Meyron-Holtz E, Smith S, Land W, Tsai RY, Berger UV, Sheng ZH, Rouault TA (2004) Expression of the iron transporter ferroportin in synaptic vesicles and the blood-brain barrier. Brain Res 1001:108-117

33. Yoon T, Cowan JA (2004) Frataxin-mediated iron delivery to ferrochelatase in the final step of heme biosynthesis. J Biol Chem 279:25943-25946 